\title{
Influência da granulometria e do teor de resíduo de polimento de placas cerâmicas na resistência mecânica de matrizes cimentícias
}

\author{
Influence of particle size distribution and \\ content of ceramic tile polishing residues \\ on mechanical performance of \\ cementitious matrices
}

Eliane Betânia Carvalho Costa ${ }^{1}$, Brunno Pereira Barros ${ }^{2}$,
Marienne do Rocio de Mello Maron da Costa

\footnotetext{
${ }^{1}$ Faculdade de Engenharia Civil - FECIV/UFU, Av. João Naves de Ávila, 2121, Campus Santa Mônica, Bloco 1Y, CEP: 38400-902, Uberlândia, MG, Brasil.

${ }^{2}$ Departamento de Engenharia Civil - DACOC/UTFPR, Rua Dep. Heitor de Alencar Furtado, 5000, Bloco A, Bairro Cidade Industrial, CEP: 81280-340, Curitiba, PR, Brasil.

${ }^{3}$ Setor de tecnologia/UFPR - Centro Politécnico, Jardim das Américas, sala 05.04, CEP: 80000-000, Curitiba, PR, Brasil. e-mail: elianebetania@ufu.br, brunno13pb@hotmail.com, mariennemaron@gmail.com
}

\section{RESUMO}

Este trabalho tem como objetivo avaliar a influência da granulometria e do teor de resíduo de polimento de placas cerâmicas em substituição ao cimento na resistência mecânica de matrizes cimentícias. Foram produzidos oito tipos de argamassa, com traço 1:3 em massa com relação água/finos de 0,48, com variações de 0 , 10, 30 e $50 \%$ de resíduo em substituição ao cimento. Foram adotados resíduos com duas granulometrias distintas: in natura e moído por 30 minutos em moinho de bolas. O resíduo foi caracterizado pelos ensaios de massa específica, granulometria a laser, difração de raios $\mathrm{X}$ e método Chapelle modificado. As argamassas foram avaliadas no estado fresco pelo índice de consistência, densidade de massa e teor de ar e, no estado endurecido, pela resistência à compressão aos 7, 28 e 150 dias. A moagem do resíduo de polimento cerâmico mostrou-se benéfica, visto que promoveu o desenvolvimento da atividade pozolânica e propiciou um aumento da resistência à compressão. Os resultados mostraram que o resíduo moído num teor de $30 \%$ propicia uma resistência à compressão de $34 \mathrm{MPa}$ aos 28 dias, podendo atingir $38 \mathrm{MPa}$ aos 150 dias. A substituição do cimento pelo resíduo de polimento com granulometria controlada pode ser favorável por apresentar menor custo e impacto ambiental, tanto pelo reaproveitamento do resíduo ou como contribuição para a mitigação do $\mathrm{CO}_{2}$.

Palavras-chave: cimento, resíduo de polimento, finos, argamassa.

\section{ABSTRACT}

This study aims to evaluate the influence of particle size distribution and the content of the replacement of cement by ceramic tile polishing residue on the mechanical strength of cementitious matrices. Eight types of mortars were produced in a ratio of 1:3 by weight (cement: sand) and water/fine (cement + residue) ratio of 0.48 with levels of replacement of $0 ; 10 ; 30$ and $50 \%$ of ceramic tile polishing residue. It was adopted waste with two different particle size distributions: in natura and ground by 30 minutes in a ball mill. Residues were characterized by specific mass; laser diffraction analysis; X-ray diffraction and Chapelle modified method. In the fresh state, mortars were evaluated by tests of consistency, mass density and air content and in the hardened state by compression strength at 7,28 and 150 days. The grinding of ceramic tile polishing residue was beneficial because it promoted the development of pozzolanic activity and an increase of the compression strength. The results showed that the replacement of $30 \%$ cement by ground residue resulted in compressive strength of $34 \mathrm{MPa}$ at 28 days and it can reach $38 \mathrm{MPa}$ at 150 days. The replacement of cement by ceramic tile polishing residue with a controlled size particle distribution can be favorable to the industry because it 
possible reduce costs and environmental impact both by reuse of the residue or as a contribution to $\mathrm{CO}_{2}$ mitigation.

Keywords: cement, polishing residue; fines; mortar

\section{INTRODUÇÃO}

A substituição do cimento por materiais alternativos tem sido uma estratégia adotada pela indústria para a redução da emissão do $\mathrm{CO}_{2}$. No Brasil, há a incorporação de filer calcário, escória de alto forno e pozolanas durante o processo de produção do cimento, em diferentes teores. No entanto, devido à limitação da disponibilidade de algumas adições, tal como cinzas volantes e escórias de alto-forno [1], outros materiais alternativos com granulometria controlada têm sido estudados ou aplicados, tais como finos calcários [2, 3] argilas calcinadas $[4,5,6]$, resíduos de polimento cerâmico $[7,8,9]$.

O uso de partículas mais finas que o cimento convencional $(<15 \mu \mathrm{m})$ ativas ou não, poderá ser uma das alternativas para reduzir a porosidade do sistema, assim como melhorar o reforço e a estabilidade dimensional de fases hidratadas, seja pelo efeito físico e/ou químico que estas exercem [10]. Além disto, as partículas finas atuam como pontos de nucleação das partículas de cimento, acelerando as reações de hidratação. Se pozolânicas, apresentam reações de hidratação mais lentas que irão consumir o hidróxido de cálcio para a produção de silicato de cálcio hidratado, principal responsável pela resistência de materiais cimentícios [11].

Durante a produção das placas cerâmicas, é necessária uma etapa de polimento para nivelar, retirar riscos e defeitos e dar brilho a superfície final. Nesta etapa, é gerada uma grande quantidade de resíduo, constituído de material cerâmico e do abrasivo e a água utilizada. Estes são depositados em lagoas de efluentes, sendo a fase líquida reaproveitada e a sólida, denominada torta de polimento armazenada em local fechado. Segundo Ramos, as indústrias do sul catarinense geram em torno de $1 \%$ de lama do processo de polimento [12] e conforme os dados da ANFACER, o Brasil produziu cerca de 792 milhões de toneladas de placas cerâmicas em 2016 [13]. Assim, pode-se supor que em 2016 foram gerados cerca de 7,92 milhões de toneladas de resíduos, uma quantidade de significativa. A reciclagem deste resíduo visa o desenvolvimento de materiais alternativos, a redução do consumo de recursos naturais, o resgate do investimento e a melhora da imagem social da empresa geradora do resíduo.

O resíduo de polimento das placas cerâmicas tem sido investigado como adição ou substituição ao cimento [8, 9, 14- 16], como agregado miúdo [7, 17]; na produção de concretos $[17,18,20]$ e de cimentos geopoliméricos $[12 ; 21 ; 22]$. AY e UNAL [14] analisaram a influência do uso de resíduo de placas cerâmicas moídas em adição ao cimento em diferentes teores $(25 \% ; 30 \% ; 35 \%$ e $40 \%)$ verificaram que este material apresentava atividade pozolânica e a utilização de teores em até $35 \%$ apresentavam resistência à compressão de 33,7 MPa.

ANDREOLA et al. [15] verificaram que argamassas produzidas com 25\% de resíduos de placas cerâmicas (porcelanato e grês) obtidos dos processos de polimento e esmaltação em substituição à massa de cimento proporcionavam uma redução da resistência mecânica nas idades iniciais e aos 28 dias em comparação a de referência. No entanto, após 90 dias, a argamassa com $25 \%$ de resíduo de polimento de porcelanato apresentou maior resistência mecânica e menor porosidade em relação a de referência. Os autores constataram por meio de análises de termogravimetria e difração de raios $\mathrm{X}$ a redução da quantidade de portlandita e a formação de silicatos de cálcio hidratado (C-S-H) e silico-aluminato de cálcio hidratado (C-A-S-H) confirmando a reação pozolânica.

STEINER et al. [9] estudando o efeito da substituição do cimento por resíduos de polimento de porcelanato e grês constataram que esse resíduo apresenta uma elevada pozolanicidade e, consequentemente, apresentam menor resistência mecânica até os 28 dias. Aos 120 dias, houve um acréscimo da resistência mecânica para as argamassas com $25 \%$ de resíduo em substituição à massa de cimento, corroborando com os resultados obtidos por ANDREOLA et al. [15].

LI et al. [16] avaliaram o desempenho mecânico e a microestrutura de argamassas produzidas com resíduo de polimento cerâmico com granulometria inferior a $1,18 \mathrm{~mm}$ e teores de substituição de até $20 \%$ em relação ao volume de pasta de cimento. Os resultados obtidos indicaram que para uma mesma relação água/cimento houve um aumento de $85 \%$ da resistência à compressão aos 7 e 28 dias com a redução de $33 \%$ do teor de cimento devido a densificação da microestrutura ocasionada pelo efeito filer do resíduo utilizado.

As pesquisas supracitadas confirmam que o uso de resíduos de placas cerâmicas apresentam potencial para aplicação na indústria cimentícia devido a sua atividade pozolânica. Embora apontem a importância da finura do resíduo, a influência desta ainda não foi avaliada. Neste sentido, o presente trabalho tem como objetivo avaliar a influência da granulometria e do teor de resíduo de polimento de placas cerâmicas em substituição ao cimento no desempenho mecânico de matrizes cimentícias. 


\section{MATERIAIS E MÉTODOS}

A presente pesquisa foi desenvolvida com o objetivo de verificar o efeito da incorporação de resíduo de polimento de placas cerâmicas em substituição ao cimento no desempenho mecânico de argamassas. O resíduo de polimento cerâmico foi obtido pela empresa Portobello S.A, sendo oriundo da torta de polimento. Este encontrava-se armazenado em ambiente fechado, e ao chegar ao laboratório foi feita a secagem do mesmo em estufa a $100 \pm 5^{\circ} \mathrm{C}$ para eliminação da umidade residual (37\%). O resíduo foi utilizado em duas granulometrias, a saber: (a) in natura (RPN) - obtido após a secagem em estufa sem qualquer tratamento prévio, e (b) moído (RPM) - o resíduo após a secagem foi submetido a moagem por 30 minutos em moinho cerâmico com esferas de diâmetros e massas distintas. Para evitar a aglomeração de partículas, antes da moagem foi borrifado etileno glicol na superfície do moinho e das bolas. A massa de resíduo foi mantida constante e igual a $2 \mathrm{~kg}$ para cada ciclo de moagem. Após moagem, o material foi peneirado em peneira de malha $150 \mu \mathrm{m}$ e utilizada as partículas passantes nessa peneira. Para ambos os tipos de resíduo foram analisados os teores de $0,10,30$ e $50 \%$ em substituição a massa de cimento na produção de argamassas, conforme procedimentos da NBR 7215 [23].

\subsection{Caracterização dos materiais: cimento e resíduo de polimento}

O cimento e o resíduo de polimento foram caracterizados por meio de ensaios de massa específica [24] e de granulometria. A distribuição granulométrica do resíduo in natura foi feita por meio de peneiramento mecânico, conforme recomendações da NBR NM 248 [25] e a das partículas de cimento e do resíduo moído medida por difração a laser devido ao tamanho das partículas. Para tal, utilizou-se equipamento Malvern Mastersizer 2000, com acessório Scirocco 2000 realizado via seca.

Para verificação da atividade pozolânica do resíduo de polimento foi realizado o ensaio de Chapelle segundo método descrito na NBR 15895 [26]. O ensaio consistiu em manter sob agitação uma mistura de $1 \mathrm{~g}$ de resíduo de polimento e $2 \mathrm{~g}$ de óxido de cálcio em $250 \mathrm{~mL}$ de água por um período de $16 \pm 2$ horas, em temperatura de $(90 \pm 5)^{\circ} \mathrm{C}$ em frasco Erlenmeyer. Em seguida, a solução foi resfriada em água corrente até atingir a temperatura ambiente. Então, adicionou-se $250 \mathrm{~mL}$ de solução de sacarose $(250 \mathrm{~g} / \mathrm{L})$ e fez-se a agitação durante cerca de 15 min, empregando-se agitador rotativo. Foi feita a titulação com solução de $\mathrm{HCl}$ 0,1M, empregando-se como indicador uma solução de fenolftaleína $(1 \mathrm{~g} / \mathrm{L})$. O índice de atividade pozolânica foi obtido pela quantidade de hidróxido de cálcio fixado por grama de resíduo de polimento analisado.

A análise mineralógica das amostras de cimento e resíduo de polimento foram realizadas por difratometria de raios X, utilizando difratômetro Shimadzu XRD-7000, com radiação Cu, $40 \mathrm{kV}$ e $40 \mathrm{~mA}$. A análise foi realizada no intervalo $2 \theta^{\circ}=5-120$, com passo angular $0,02^{\circ} 2 \theta^{\circ}$ e tempo de passo $0,60 \mathrm{~s}$. Para a realização deste ensaio, foi utilizado a amostra moída para facilitar a compactação do pó.

A caracterização química do cimento e do resíduo foram fornecidas pelos respectivos fabricantes, cujos resultados estão apresentados na Tabela 1.

Tabela 1: Caracterização química do cimento e resíduo de polimento cerâmico estudados.

\begin{tabular}{c|c|c}
\hline ÓXIDOS (\%) & CIMENTO $^{(\mathbf{A})}$ & RESÍDUO DE POLIMENTO $^{(\mathbf{A})}$ \\
\hline $\mathrm{Al}_{2} \mathrm{O}_{3}$ & 4,32 & 18,93 \\
\hline $\mathrm{SiO}_{2}$ & 18,99 & 65,78 \\
\hline $\mathrm{Fe}_{2} \mathrm{O}_{3}$ & 2,74 & 1,27 \\
\hline $\mathrm{CaO}$ & 61,16 & 1,26 \\
\hline $\mathrm{MgO}$ & 4,49 & 2,25 \\
\hline $\mathrm{SO}_{3}$ & 2,94 & - \\
\hline $\mathrm{TiO}_{2}$ & - & 0,49 \\
\hline $\mathrm{ZrO}_{2}$ & 0,49 \\
\hline $\mathrm{P}_{2} \mathrm{O}_{5}$ & - & 0,10 \\
\hline $\mathrm{BaO}$ & - & 0,06 \\
\hline $\mathrm{ZnO}$ & - & 0,13 \\
\hline $\mathrm{CaO}$ livre & - & - \\
\hline Perda ao fogo & 0,81 & 3,80 \\
\hline Resíduo insolúvel & 3,27 & - \\
\hline
\end{tabular}




\begin{tabular}{c|c|c}
\hline Equivalente alcalino $^{(\mathrm{b})}$ & 0,69 & 4,18 \\
\hline
\end{tabular}

Os resultados indicam que o resíduo de polimento é constituído principalmente por sílica $(65,78 \%)$ e alumínio $(18,93 \%)$, com pequena quantidade de sais solúveis $(4,18 \%)$. Esse resultado foi similar ao obtido por $[9,14,15]$. Também foi verificado alguns traços de zircônio, titânio e bário provenientes do processo de fabricação.

\subsection{Matrizes cimentícias: composição e produção}

Foram produzidos oito tipos de argamassa, com traço 1:3 em massa com relação água/finos (cimento + resíduo) de 0,48, com variações de 0,10,30 e 50\% de resíduo em substituição ao cimento, e com granulometria distinta do resíduo (in natura e após moagem). Foi utilizado cimento de alta resistência inicial (CPV - ARI), por apresentar menor quantidade de adições minerais em sua composição; areia normalizada do IPT em quatro frações: grossa, média grossa, média fina e fina; água da rede de abastecimento público da cidade de Curitiba; além dos resíduos supracitados.

A mistura das argamassas foi realizada em misturador mecânico/elétrico com motor de 3HP, trifásico com duas velocidades sendo $140 \mathrm{rpm}$ em baixa e $285 \mathrm{rpm}$ em alta rotação conforme os procedimentos descritos pela NBR 7215 [23]. Para a mistura, os finos (cimento e resíduo) juntamente com a água foram colocados no misturador e agitados por $30 \mathrm{~s}$ em velocidade baixa. Em seguida, acrescentou-se areia, previamente com as frações homogeneizadas, sem paralisar a mistura, colocou-se em velocidade alta por $30 \mathrm{~s}$. Desligou-se o equipamento e esperou-se por $75 \mathrm{~s}$. Por fim, misturou-se por mais $60 \mathrm{~s}$ em velocidade alta.

\subsection{Ensaios realizados}

Após a mistura das argamassas foram realizados os ensaios de densidade de massa, do teor de ar incorporado [27] e do índice de consistência [28]. Para avaliação das propriedades das argamassas no estado endurecido foram moldados seis corpos de prova cilíndricos com diâmetro igual a $50 \mathrm{~mm}$ e espessura de $100 \mathrm{~mm}$ moldados em quatro camadas em mesa de queda e aplicados 30 quedas por camada. As superfícies foram niveladas com espátula. Os corpos de prova ficaram em ambiente úmido entre 20 e $24 \mathrm{~h}$. Após esse período, os moldes foram retirados e os corpos de prova imersos em água saturada de cal até a realização do ensaio de resistência à compressão. Os ensaios foram realizados aos 7, 28 e 150 dias utilizando prensa hidráulica EMIC DL 3000, conforme os procedimentos da NBR 5739 [29].

\subsection{Eficiência do uso de ligante}

A eficiência do uso do ligante foi medida pelo indicador $I L$ - índice de ligante, proposto por DAMINELI et al. [30]. Este índice que mede a quantidade necessária de ligante para obtenção de uma unidade de determinado parâmetro de desempenho (Equação 1):

$$
\mathrm{IL}=\frac{\mathrm{b}}{\mathrm{p}}
$$

em que b é o consumo total de ligante do material em $\mathrm{kg} / \mathrm{m}^{3}$ e p é o requisito de desempenho. Neste trabalho, será medido a quantidade de ligante necessária para a obtenção de $1 \mathrm{MPa}$ de resistência à compressão aos 28 dias.

\subsection{Análise estatística}

Os resultados de resistência à compressão obtidos foram submetidos a análise de variâncias (ANOVA) a um nível de probabilidade de 5\%. Após a conclusão de que existe diferença significativa entre os tratamentos, por meio do teste $\mathrm{F}$, a magnitude destas diferenças foi avaliada utilizando teste de comparações múltiplas teste de Duncan. Esse teste permite comparar grupos entre si, com base na diferença mínima significativa $(d m s)$. A estatística do teste é dada pela Equação 2:

$$
d m s=q_{i} \sqrt{\frac{s_{e}^{2}}{n}}\left(\frac{1}{r_{1}}+\frac{1}{r_{2}}+\cdots+\frac{1}{r_{n}}\right)
$$

em que $q_{i}$ é valor tabelado por Duncan obtido em função da distância entre as médias comparadas $(i=\mathrm{p}+n$; $\mathrm{p}=$ número de médias localizadas entre o número de tratamentos que estão sendo comparados $(n)$, relacionadas em ordem crescente; $s_{e}$ é o número de graus de liberdade do resíduo; e, $r$ é o número de observações variável. 
Os cálculos da ANOVA e teste de Duncan foram realizados com auxílio do programa Statistica 9.0 da Statsoft ${ }^{\circledR}$.

\section{RESULTADOS E DISCUSSÃO}

\subsection{Caracterização dos materiais}

A massa específica do cimento e do resíduo de polimento foram iguais a $3,14 \mathrm{~g} / \mathrm{cm}^{3}$ e $2,56 \mathrm{~g} / \mathrm{cm}^{3}$, respectivamente. Os resultados da distribuição granulométrica do cimento e dos resíduos estudados estão apresentados na Figura 1. Observa-se que o resíduo in natura apresenta uma distribuição polimodal, com picos máximos em $150 \mu \mathrm{m}$ e $1200 \mu \mathrm{m}$. Esses valores indicam que o resíduo in natura apresenta maior granulometria em relação ao cimento e o resíduo moído. O resíduo moído apresentou diâmetro médio de 7,41 $\mu \mathrm{m}$ e o cimento de $12,16 \mu \mathrm{m}$. O cimento apresenta $90 \%$ das partículas inferiores a $36 \mu \mathrm{m}$ enquanto o resíduo moído $42 \mu \mathrm{m}$. Estes resultados indicam que a moagem foi eficiente em reduzir o tamanho das partículas do resíduo de polimento.

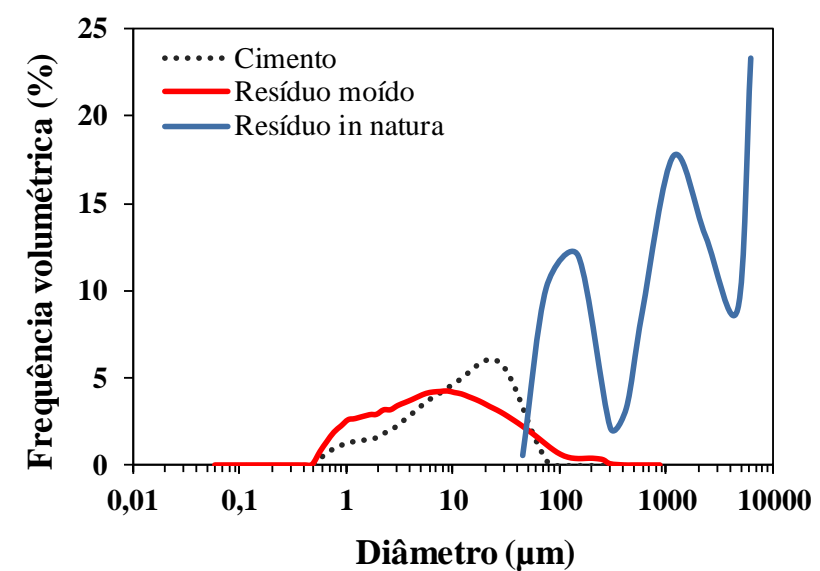

Figura 1: Distribuição granulométrica do cimento e resíduos de polimento utilizados na produção das argamassas.

A difração de raios $\mathrm{X}$ do resíduo de polimento (Figura 2) mostra que as fases predominantes foram o quartzo $\left(\mathrm{SiO}_{2}\right)$, a muscovita $\left[\mathrm{KAl}_{2}\left(\mathrm{Si}_{3} \mathrm{AlO}_{10}\right)(\mathrm{OH})_{2}\right]$, a caulinita $\left.\left[\mathrm{Al}_{2} \mathrm{Si}_{2} \mathrm{O}_{5}\right)(\mathrm{OH})_{4}\right]$ e a dolomita $\left[\mathrm{CaMg}\left(\mathrm{CO}_{3}\right)_{2}\right]$, tal resultado corresponde com a análise química fornecida pelo fabricante que indicou a presença de $66 \%$ de sílica, $19 \%$ de alumina e 2,3\% de dolomita. Na Figura 2 também pode-se observar a presença acentuada de picos cristalinos e a de um halo amorfo discreto entre $18^{\circ}$ e $36^{\circ}$ indicando a reatividade do resíduo de polimento. Tal resultado corrobora com os obtidos por Steiner, BERNADIM e PELISSER [9].

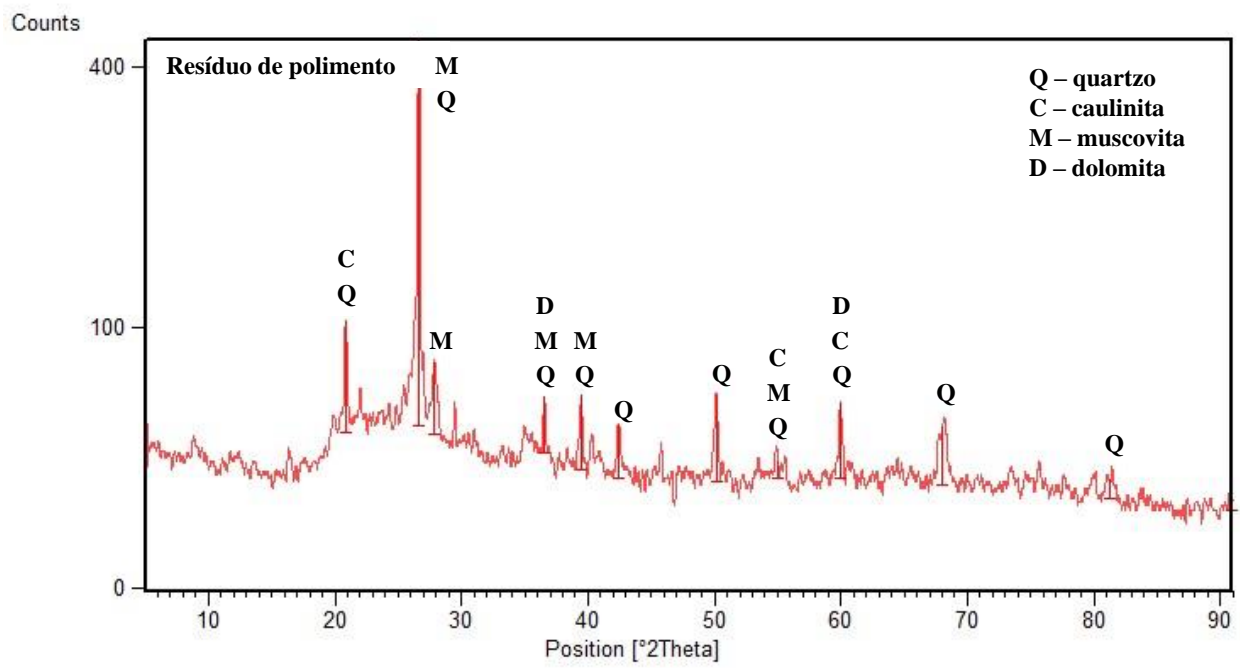

Figura 2: Difratograma do resíduo de polimento cerâmico. 
Na Figura 3 está apresentado os teores de cal fixados pelos resíduos de polimento obtidos conforme as especificações da NBR 15895 [26]. Observa-se que há uma diferença entre o comportamento do resíduo natural e o moído. O resíduo de polimento moído apresentou maior reatividade em relação ao in natura, com consumo de $406 \mathrm{mg} \mathrm{CaO} / \mathrm{g}$ de adição. Tal resultado indica que o resíduo de polimento moído pode ser classificado como material pozolânico conforme índice mínimo de $330 \mathrm{mg} / \mathrm{de} \mathrm{CaO} / \mathrm{g}$ de amostra estabelecido por RAVERDI et al. [31].

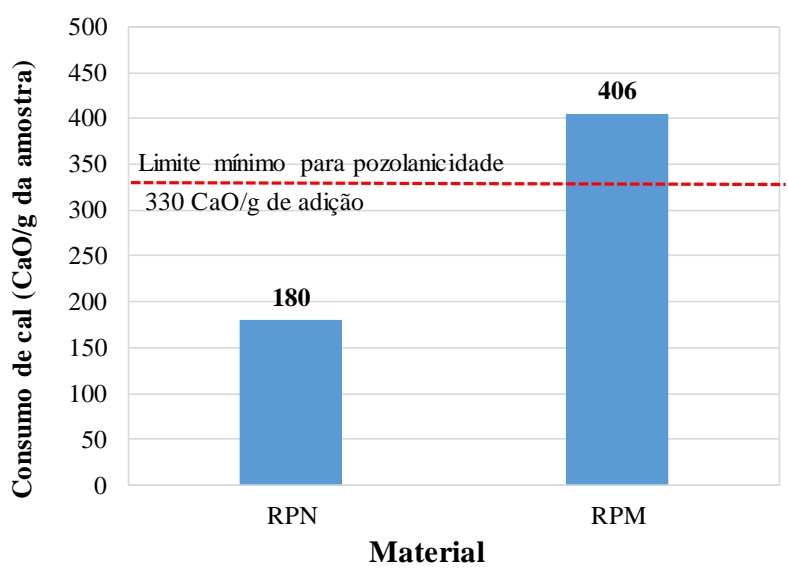

Figura 3: Resultados do ensaio de pozolanicidade pelo método de Chapelle para os diferentes tipos de resíduo de polimento estudados: RPN - resíduo de polimento natural; RPM - resíduo de polimento moído.

ANDREOLA et al. [15] por meio de ensaios de difração de raios X e análise de termogravimetria constataram a atividade pozolânica de um resíduo de polimento de porcelanato com partículas de diâmetro médio equivalente a $7 \mu \mathrm{m}$. A atividade pozolânica de resíduo de polimento de porcelanato também foi constatada por Steiner, Bernadim e Pelisser por meio de difração de raios X para partículas de resíduo com diâmetro médio variando entre 6 e $13 \mu \mathrm{m}$ [9]. Os resultados obtidos neste trabalho comprovam a importância da finura do resíduo para o desenvolvimento da atividade pozolânica.

\subsection{Propriedades das argamassas}

As propriedades das argamassas no estado fresco estão apresentadas na Tabela 2.

Tabela 2: Propriedades das argamassas produzidas no estado fresco.

\begin{tabular}{|c|c|c|c|c|c|c|}
\hline $\begin{array}{c}\text { ARGAMAS- } \\
\text { SA }\end{array}$ & $\begin{array}{c}\text { TEOR DE } \\
\text { RESÍDUO } \\
(\%) \\
\end{array}$ & $\begin{array}{l}\text { TIPO DE } \\
\text { RESÍDUO }\end{array}$ & $\begin{array}{c}\text { CONSUMO DE } \\
\text { CIMENTO } \\
\left(\mathrm{kg} / \mathrm{m}^{3}\right) \\
\end{array}$ & $\begin{array}{c}\text { ÍNDICE DE } \\
\text { CONSISTÊNCIA } \\
(\mathrm{mm})\end{array}$ & $\begin{array}{c}\text { DENSIDADE } \\
\left(\mathrm{kg} / \mathrm{m}^{3}\right)\end{array}$ & $\begin{array}{c}\text { TEOR DE } \\
\text { AR (\%) }\end{array}$ \\
\hline REF & 0 & - & 401,79 & 195 & 1800 & 21,71 \\
\hline RPN10 & 10 & in natura & 353,57 & 200 & 1760 & 23,18 \\
\hline RPM10 & 10 & moído & 355,58 & 180 & 1770 & 22,75 \\
\hline RPN30 & 30 & in natura & 278,13 & 170 & 1780 & 21,78 \\
\hline RPM30 & 30 & moído & 281,25 & 165 & 1800 & 20,90 \\
\hline RPN50 & 50 & in natura & 190,85 & 180 & 1710 & 24,34 \\
\hline RPM50 & 50 & moído & 199,78 & 155 & 1790 & 20,80 \\
\hline
\end{tabular}

Observa-se que a argamassa RPN10 apresentou maior índice de consistência em relação as demais argamassas. Isso pode ser explicado pela granulometria do material que apresenta partículas superiores em comparação ao cimento e ao resíduo moído. No entanto, de modo geral, verificou-se que houve uma redução da consistência das argamassas com o incremento do teor de resíduo de polimento (30\% e 50\%), sendo mais intensa com o resíduo moído. Isso ocorre porque com o acréscimo de finos no sistema há um aumento da área superficial, o que irá demandar um maior teor de água para manter a mesma consistência de uma arga- 
massa sem finos. O acréscimo de finos também proporciona uma diminuição do teor de ar da argamassa no estado fresco devido a uma melhoria do empacotamento de partículas no sistema [32].

Andreola et al. analisando a consistência das argamassas por meio do ensaio de mini abatimento com o uso de $25 \%$ de resíduo de polimento também verificou uma redução da trabalhabilidade em comparação com a argamassa sem resíduo. Segundo os autores, essa diminuição ocorre devido a forma das partículas e a tendência de aglomeração dos finos [15].

Na Figura 4 estão apresentados os resultados de resistência à compressão aos 7, 28 e 150 dias obtidos para as argamassas estudadas.

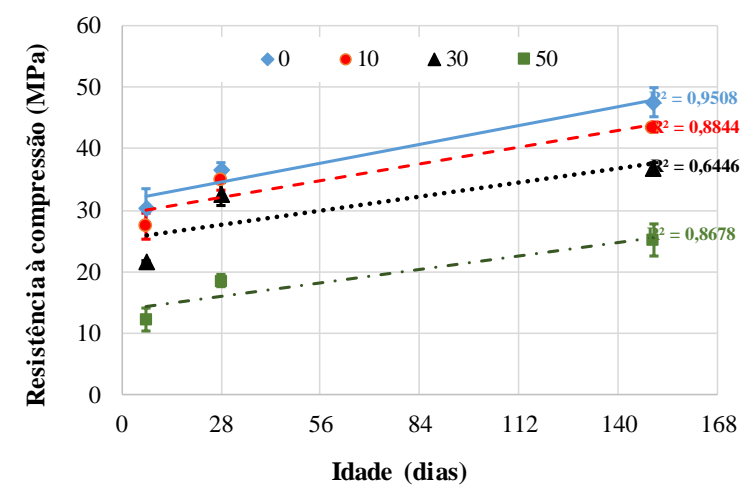

(a) Resíduo de polimento in natura - RPN

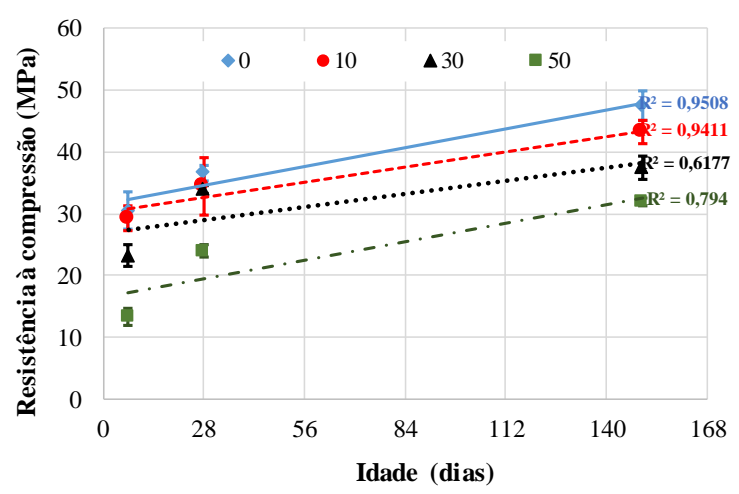

(b) Resíduo de polimento moído - RPM

Figura 4: Resistência à compressão em função da idade e do teor de resíduo de polimento (a): RPN - resíduo de polimento in natura; (b) RPM - resíduo de polimento moído.

Nota-se que houve uma redução dos valores médios da compressão das argamassas com o acréscimo do teor de resíduo de polimento utilizado em substituição ao cimento em relação a argamassa de referência (REF) em todas as idades. Aos 7 dias, a porcentagem de redução foi de 10\%, 29\% e 60\% para as argamassas RPN10; RPN 30 e RPN50, respectivamente. Para as argamassas RPM10, RPM30, RPM 50, a redução foi de $-5 \% ; 24 \%$ e $57 \%$. Com o avanço da idade, a porcentagem de redução diminuiu em relação a argamassa de referência para ambos os resíduos, sendo que a menor dimensão das partículas do resíduo moído contribui para uma menor taxa de redução.

Resultados similares foram obtidos por Steiner, BERNADIM e PELISSER [9] ao analisarem a substituição de cimento por resíduo de polimento em teores de $10 \% ; 20 \% ; 25 \%$; $30 \%$ e $40 \%$. Essa redução da resistência à compressão pode ser explicada devido ao efeito de diluição, pois com a redução do consumo de cimento há uma diminuição da capacidade ligante entre as partículas. Além disso, por haver um aumento da relação água/cimento, a adição de partículas do resíduo irá diminuir o volume de produtos hidratados, o que para um teor de água constante implica num aumento da porosidade do sistema, com consequente queda de resistência. O resíduo de polimento quando moído diminui esse efeito, pois os grãos menores contribuem para o empacotamento das partículas no sistema. Além disso, o efeito pozolânico verificado neste material também pode ter contribuído por apresentar melhor resistência à compressão em relação ao resíduo in natura. Ensaios adicionais para comprovação da atividade pozolânica pela alteração de produtos hidratados com uso desse resíduo precisam ser realizados.

Verifica-se também que a taxa de desenvolvimento de resistência para as argamassas foi mais efetiva entre 7 e 28 dias. Para as argamassas produzidas com 10\% de resíduo de polimento, a taxa foi equivalente a $21 \%$ aos 28 dias e $25 \%$, aos 150 dias. Para este teor de resíduo, a granulometria do resíduo não influenciou no ganho de resistência. A substituição do cimento por $30 \%$ de resíduo propiciou maior taxa de desenvolvimento de resistência aos 28 dias, sendo o acréscimo mais efetivo para o resíduo moído (46\%) em relação ao in natura (34\%). Entre 28 dias e 150 dias, ambos resíduos apresentaram uma taxa equivalente a $11 \%$.

De acordo com Lawrence, CYR e RINGOT [33] quando adicionados a uma matriz cimentícia, as partículas finas também podem atuar como agente de nucleação, participando da ativação química do cimento. Segundo os autores, há uma redução da espessura da camada dos compostos hidratados formados na superfície dos grãos de cimento, acelerando o desenvolvimento da hidratação e, consequentemente, o aumento da resistência inicial. No caso das argamassas estudadas, o efeito de diluição devido ao aumento do teor de resí- 
duo foi mais evidente para a redução da resistência em comparação a argamassa de referência. No entanto, ao comparar a granulometria do resíduo moído observa-se que o resíduo de polimento moído apresentou maior resistência em todas as idades, sendo que o desenvolvimento da mesma foi mais pronunciado dos 7 aos 28 dias. A granulometria do resíduo contribuiu para minimizar os efeitos de diluição, quanto menor a granulometria do resíduo maior a resistência à compressão.

O teor de substituição do cimento pelo resíduo de polimento bem com a idade de avaliação das argamassas interfere significativamente nos valores de resistência à compressão como mostra os resultados da ANOVA apresentados na Tabela 3. De acordo com o teste de Duncan, utilizado a comparação do efeito do teor de resíduo de polimento, verificou-se que os teores analisados propiciam resistência à compressão distintas entre si.

Tabela 3: Análise de variância do efeito do teor de resíduo e da idade de avaliação na resistência à compressão das argamassas estudadas.

\begin{tabular}{c|c|c|c|c|c|c}
\hline FONTE DE VARIAÇÃO & SQ & GL & MQ & FCAL & FTAB & RESULTADO \\
\hline Teor de residuo (\%) & 4905,11 & 3 & 1635,04 & 116,15 & 2,71 & Significativo \\
\hline Idade (días) & 3924,96 & 2 & 1962,48 & 139,42 & 3,11 & Significativo \\
\hline Erro & 1168,34 & 83 & 14,08 & - & - & - \\
\hline Total & 9998,40 & 88 & - & - & - & - \\
\hline
\end{tabular}

Legenda: SQ - soma dos quadrados; GL - graus de liberdade; MQ - média dos quadrados; F - parâmetro de Fisher para o teste de significância dos efeitos.

Na Figura 5 está apresentada o índice de ligante das argamassas produzidas, calculado conforme proposto por DAMINELI et al. [30]. Este índice $\left(i_{L}\right)$ mede a quantidade necessária de ligante para obtenção de uma unidade de determinado parâmetro de desempenho, neste caso a quantidade de necessária de ligante para a obtenção de $1 \mathrm{MPa}$ de resistência à compressão aos 28 dias.

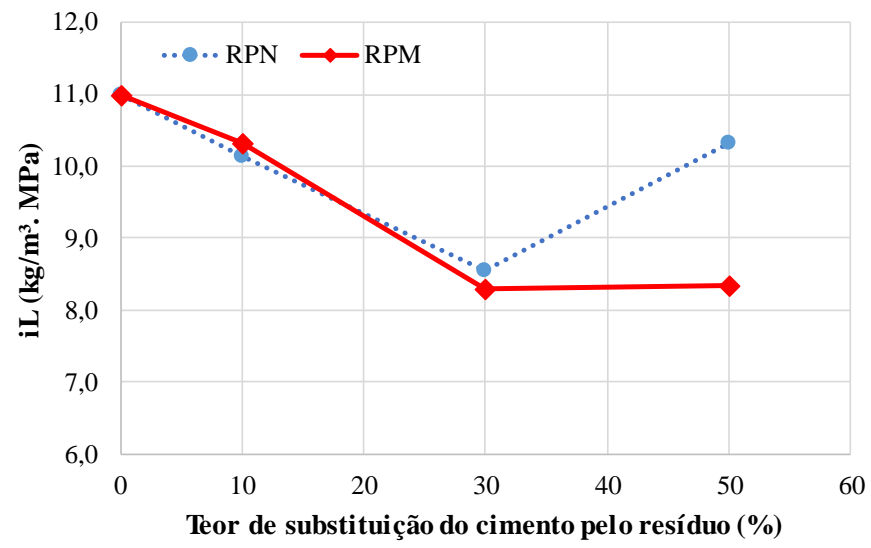

Figura 5: Índice de ligante em função do teor de substituição do cimento pelos resíduos de polimento cerâmico aos 28 dias.

A argamassa de referência apresenta um índice de $11 \mathrm{~kg} / \mathrm{m}^{3} . \mathrm{MPa}$. A utilização do resíduo de polimento propiciou uma redução desse índice para todas as argamassas. O melhor desempenho foi obtido utilizando o resíduo moído no teor de $30 \%$ ( $\mathrm{IL}=8,3$ ). Isso indica que a redução da granulometria do resíduo através da moagem propiciou um melhor desempenho do sistema. Steiner, BERNADIM e PELISSER [9] também constataram uma melhoria do índice de eficiência do ligante ao utilizar 30\% de resíduo cerâmico de polimento em substituição ao cimento.

Os resultados mostraram que o resíduo moído num teor de $30 \%$ propicia uma resistência à compressão de $34 \mathrm{MPa}$ aos 28 dias, podendo atingir $38 \mathrm{MPa}$ aos 150 dias. Desse modo, a substituição do cimento pelo resíduo de polimento pode ser favorável à indústria, com custo mais baixo e menor impacto ambiental, tanto pelo reaproveitamento do resíduo como pela mitigação do $\mathrm{CO}_{2}$. 


\section{CONCLUSÕES}

O efeito do teor de substituição do cimento por resíduos de placas cerâmicas na resistência à compressão de matrizes cimentícias foi investigado. O resíduo foi avaliado em duas granulometrias distintas: in natura com partículas variando entre $40 \mu \mathrm{m}$ e $2000 \mu \mathrm{m}$ e o moído com $90 \%$ das partículas com diâmetro inferior a $42 \mu \mathrm{m}$. Foram avaliados os teores de $0 \%, 10 \%, 30 \%$ e $50 \%$ de substituição do cimento por resíduos em argamassas com traço 1:3 (cimento: areia normatizada) e relação água/finos (cimento + resíduo). A partir dos resultados experimentais e discussões acima, as seguintes conclusões podem ser extraídas:

- O resíduo de polimento moído apresentou um consumo de $405 \mathrm{mg} \mathrm{CaO} / \mathrm{g}$ de adição, indicando a pozolanicidade. O mesmo não foi observado para o resíduo de polimento in natura mostrando a importância da finura do material para o desenvolvimento da atividade pozolânica.

- A resistência mecânica das argamassas diminui com o aumento do teor de substituição do cimento pelo uso de resíduo de polimento devido ao efeito de diluição. A finura e a distribuição granulométrica das partículas do sistema são importantes. A substituição do ligante pelo resíduo moído resultou em resistência à compressão superior à do resíduo de polimento in natura, principalmente nos teores mais elevados.

- A substituição do cimento pelo resíduo de polimento pode ser favorável à indústria, com custo mais baixo e menor impacto ambiental, tanto pelo reaproveitamento do resíduo como pela mitigação do $\mathrm{CO}_{2}$. O resíduo moído num teor de $30 \%$ propicia uma resistência à compressão de $34 \mathrm{MPa}$ aos 28 dias, podendo atingir $38 \mathrm{MPa}$ aos 150 dias e melhor índice de eficiência do ligante.

\section{AGRADECIMENTOS}

Os autores gostariam de agradecer a empresa Cimento Itambé e ao Eng. Alécio Mattana pela doação dos materiais utilizados nesta pesquisa; ao técnico Gustavo Macioski pela realização do ensaio Chapelle e ao estagiário Adriano Vasconcelos pela colaboração na caracterização dos materiais. Ao Laboratório do Núcleo de Pesquisas Tecnológicas - UTFPR, coordenado pelos Prof. José Alberto Cerri e Profa. Marcia Silva de Araújo, por ceder o moinho de bolas cerâmicos para moagem do resíduo. Ao Centro Multiusuário de Caracterização de Materiais - CMCM da UTFPR-CT pela realização dos ensaios de difração de raios X.

\section{BIBLIOGRAFIA}

[1] DAMINELI, B. L., JOHN, V. M., "Developing Low CO2 Concretes: Is Clinker Replacement Sufficient? The Need of Cement Use Efficiency Improvement”, Key Engineering Materials, v. 517, pp. 342-351, 2012.

[2] INGRAM, K., DAUGHERTY, K., "A review of limestone additions to Portland cement and concrete". Cement Concrete Composites, v.13, pp.165-170, (1991).

[3] CAMILETTI, J., SOLIMAN, A. M., NEHDI, M. L., "Effects of nano- and micro-limestone addition on early-age properties of ultra-high-performance concrete", Materials and Structures, v. 46, n. 6, pp. 881-898, Jun. 2013

[4] PAIVA, H., VElOSA, A., CACHIM, P., FERREIRA, V. M., "Effect of metakaolin dispersion on the fresh and hardened state properties of concrete", Cement and Concrete Research, v. 42, pp. 607-612, 2012.

[5] ANTONI, M., ROSSEN, J., MARTIRENA, F., et al., "Cement substitution by a combination of metakaolin and limestone", Cement and Concrete Research, v. 42, pp. 1579-1589, 2012.

[6] NEHDI, M. L., "Clay in cement-based materials: Critical overview of state-of-the-art". Construction and Building Materials, v.51, pp. 372-382, 2014.

[7] PAIXÃO, C. A., CAETANO, L. F., COLIANTE, J. G. R., et al., "Estudo da viabilidade de utilização de resíduos cerâmicos para confecção de argamassas", Cerâmica Industrial, v.16, n. 4, pp. 0, 2011.

[8] PELISSER, F., STEINER, L. R., BERNADIM, A. M., "Recycling of porcelain tile polishing residue in Portland cement hydration efficiency". Environment Science Technology, v.46, pp. 2368-2374, 2012.

[9] STEINER, L. R., BERNADIM, A. M., PELISSER, F., "Effectiveness of ceramic tile polishing residues as supplementary cementitious materials for cement mortars", Sustainable Materials and Technologies, v. 4, pp. 30-35, 2015.

[10] OLLIVIER, J. P.; MASO, J. C.; BOURDETTE, B., "Interfacial transition zone in concrete", Advanced Cement Based Materials, n. 2, pp. 20-30, 1995.

[11] MALHOTRA, V. M., MEHTA, P. K., Pozzolanic and cementitious materials, 1ed., Amsterdam, Gordon and Breach, 1996. 
[12] RAMOS, G. A., PELISSER, F., GLEIZE, P. J. P., et al., "Cimento geopolimérico contendo resíduo do polimento de placas cerâmicas", In: $59^{\circ}$ Edição Congresso Brasileiro do Concreto, Bento Gonçalves, Rio Grande do Sul, BRA, 2017.

[13] ASSOCIAÇÃO NACIONAL DOS FABRICANTES DE CERÂMICA PARA REVESTIMENTOS, LOUÇAS SANITÁRIAS E CONGÊNERES - ANFACER. Panorama: principais produtores mundiais 2016. Disponível em: < https://www.anfacer.org.br/mundial>. Acesso em: 08.02.2019.

[14] AY, N., UNAL, M., "The use of waste ceramic tile in cement production", Cement and Concrete Research, v. 30, pp. 497-499, 2000.

[15] ANDREOLA, F., BARBIERI, L., LANCELOTTI, I., et al., "New blended cement from polishing and glazing ceramic sludge", International. Journal Applied Ceramic Technology, v.7, pp. 546-555, 2010.

[16] LI, L. G., ZHUO, J., ZHUO, Y., et al., "Reutilizing ceramic polishing waste as powder filler in mortar to reduce cement content by 33\% and increase strength by 85\%", Powder Technology, v. 355, pp. 119-126, 2019.

[17] BREITENBACH, S. B., SANTOS, O. C., ANDRADE, J. C. S., et al., "Adição de resíduo do polimento de porcelanato em argamassas de restauro à base de cal”, Cerâmica, v. 63, n. 367, pp. 395-401, 2017.

[18] PACHECO-TORGAL, F., JALALI, S., "Reusing ceramic wastes in concrete", Construction and Building Materials. v.24, pp.832-838, 2010.

[19] PENTEADO, C.S.G., CARVALHO, E. V., LINTZ, R. C. C., "Reusing Ceramic Tile Polishing Waste in Paving Block Manufacturing", Journal of Cleaner Production, v. 112, p. 514-520, 2015.

[20] MATOS, P. R., OLIVEIRA, A.L., PELISSER, F., PRUDÊNCIO JR., L. R., "Rheological behavior of Portland cement pastes and self-compacting concretes containing porcelain polishing residue", Construction and Building Materials, v. 175, pp. 508-518, 2018.

[21] PINTO, E. N. M. G., "Adição de resíduo cerâmico em pastas geopoliméricas para cimentação de poços de petróleo", Tese de D. Sc., Universidade Federal do Rio Grande do Norte, Natal, 2011, 94 p.

[22] RAMOS, G. A., PELISSER, F., GLEIZE, P. J. P., et al., "Effect of porcelain tile polishing residues on geopolymer cement", Journal of Cleaner Production, v. 191, pp. 297-303, 2018.

[23] ASSOCIAÇÃO BRASILEIRA DE NORMAS TÉCNICAS. NBR 7215. Cimento Portland - Determinação da resistência à compressão. Rio de Janeiro, 2011.

[24] ASSOCIAÇÃO BRASILEIRA DE NORMAS TÉCNICAS. NBR 16605. Cimento Portland e outros materiais em pó - Determinação da massa específica. Rio de Janeiro, 2017.

[25] ASSOCIAÇÃO BRASILEIRA DE NORMAS TÉCNICAS. NBR NM 248. Agregados - Determinação da composição granulométrica. Rio de Janeiro, 2003.

[26] ASSOCIAÇÃO BRASILEIRA DE NORMAS TÉCNICAS. NBR 15895. Materiais pozolânicos - Determinação do teor de hidróxido de cálcio fixado - Método Chapelle modificado. Rio de Janeiro, 2010.

[27] ASSOCIAÇÃO BRASILEIRA DE NORMAS TÉCNICAS. NBR 13278. Argamassa para assentamento e revestimento de paredes e tetos - Determinação da densidade de massa e do teor de ar incorporado. Rio de Janeiro, 2005.

[28] ASSOCIAÇÃO BRASILEIRA DE NORMAS TÉCNICAS. NBR 13278. Argamassa para assentamento e revestimento de paredes e tetos - Determinação do índice de consistência. Rio de Janeiro, 2016.

[29] ASSOCIAÇÃO BRASILEIRA DE NORMAS TÉCNICAS. NBR 5739. Concreto - Ensaios de compressão de corpos de prova cilíndricos. Rio de Janeiro, 2007.

[30] DAMINELI, B. L., KEMEID, F. M., AGUIAR, P. S., JOHN, V. M., "Measuring the ecoefficiency of cement use". Cement and Concrete Composites, v. 32, pp.555-562, 2010.

[31] RAVERDY, M., BRIVOT, F., PAILLÈRE, A. M., DRON, R., "Appréciation de l'activité pouzzolanique des constituants secondaires", In: 7e Congrés International de la Chimie des Ciments, v. III-4. Paris, 1980.

[32] OLIVEIRA, I. R., STUDART, A. R., PILEGGI, R. G., et al., Dispersão e empacotamento de partículas: princípios e aplicações em processamento cerâmico., 1 ed., São Paulo, Fazendo Arte Editorial, 2000.

[33] LAWRENCE, P., CYR, M., RINGOT, E., "Mineral Admixtures in Mortars: effect of inert materials on short-term hydration", Cement and Concrete Research, v. 33, n. 12, pp. 1939-1947, 2003. 
COSTA, E.B.C.; BARROS, B.P.; COSTA, M.R.M.M. revista Matéria, v.25, n.4, 2020.

ORCID

Eliane Betânia Carvalho Costa

https://orcid.org/0000-0002-3692-3806

Brunno Pereira Barros

https://orcid.org/0000-0001-7253-8748

Marienne do Rocio de Mello Maron da Costa

https://orcid.org/0000-0002-9421-7985 